\title{
Genetic and Environmental Influences on Eating Behavior - A Study of Twin Pairs Reared Apart or Reared Together
}

\author{
Sonya J. Elder ${ }^{1}$, Michael C. Neale ${ }^{2}$, Paul J. Fuss ${ }^{1}$, Alice H. Lichtenstein ${ }^{1}$, Andrew S. Greenberg ${ }^{1}$, \\ Megan A. McCrory ${ }^{3}$, Thomas J. Bouchard, Jr. , Edward Saltzman ${ }^{1}$ and Susan B. Roberts ${ }^{*}, 1$ \\ ${ }^{1}$ Jean Mayer USDA Human Nutrition Research Center on Aging, Tufts University, Boston, Massachusetts, USA \\ ${ }^{2}$ Virginia Institute of Psychiatric and Behavioral Genetics and Department of Psychiatry, Virginia Commonwealth \\ University, Richmond, Virginia, USA \\ ${ }^{3}$ Department of Foods and Nutrition, Purdue University, 700 W. State St., West Lafayette, IN 47907, USA \\ ${ }^{4}$ Department of Psychology, University of Minnesota, Minneapolis, MN, USA
}

\begin{abstract}
This study examined the relative influence of genetic versus environmental factors on specific aspects of eating behavior. Adult monozygotic twins (22 pairs and 3 singleton reared apart, 38 pairs and 9 singleton reared together, age 18-76 years, BMI 17-43 kg/m²) completed the Three Factor Eating Questionnaire. Genetic and environmental variance components were determined for the three eating behavior constructs and their subscales using model-fitting univariate and multivariate analyses. Unique environmental factors had a substantial influence on all eating behavior variables (explaining $45-71 \%$ of variance), and most strongly influenced external locus for hunger and strategic dieting behavior of restraint (explaining $71 \%$ and $69 \%$ of variance, respectively). Genetic factors had a statistically significant influence on only 4 variables: restraint, emotional susceptibility to disinhibition, situational susceptibility to disinhibition, and internal locus for hunger (heritabilities were 52\%, 55\%, 38\% and 50\%, respectively). Common environmental factors did not statistically significantly influence any variable assessed in this study. In addition, multivariate analyses showed that disinhibition and hunger share a common influence, while restraint appears to be a distinct construct. These findings suggest that the majority of variation in eating behavior variables is associated with unique environmental factors, and highlights the importance of the environment in facilitating specific eating behaviors that may promote excess weight gain.
\end{abstract}

Keywords: Disinhibition, restraint, hunger, obesity, heritability, environment.

\section{INTRODUCTION}

Eating behavior is known to play an important role in energy regulation [1], and the eating behavior construct, disinhibition is correlated with current body mass index (BMI), prior weight gain over 20 years, and weight regain following weight loss [2-8]. However, the extent to which different eating behaviors are influenced by genes versus environmental factors remains uncertain.

Aspects of eating behavior such as dietary restraint and food preferences are known to be transmissible between family generations [9-11], but currently there are only six reports on the heritability of different eating behaviors [1217] assessed using a full or shortened version of the validated Three Factor Eating Questionnaire [18]. Moreover, estimates for the heritability of the three most widely recognized eating behavior constructs - disinhibition, restraint and hunger - vary widely even when the full Eating Inventory was used for determination. In addition, to our knowledge,

\footnotetext{
"Address correspondence to this author at the Energy Metabolism Laboratory, USDA HNRCA at Tufts University, 711 Washington St, Boston, MA 02111, USA; Tel: (617) 556-3237; Fax: (617) 556-3122;

E-mail: susan.roberts@tufts.edu
}

the heritabilities of the more recently proposed subscales of eating behavior have not been estimated. These subscales were proposed to identify specific aspects of eating behavior, and although low heritability estimates do not necessarily indicate high malleability of a trait, subconstructs with low heritability and/or high environmental transmission estimates may potentially have greater utility in informing effective targets for behavior change. Previous reviews have recognized the general lack of data in this area and have emphasized the need for much more research [19].

The objectives of this study were to quantify the genetic, common environmental and unique environmental influences on eating behavior constructs and subscales assessed by the Three Factor Eating Questionnaire, and to determine the extent to which genetic and environmental influences are shared by restraint, disinhibition, and hunger. As described elsewhere [20] data was collected as part of the Tufts Twin Study. MZA twin pairs constitute a particularly valuable group for assessing trait heritability because although they are extremely rare they provide substantial statistical power to estimate heritability compared to studies using other types of populations [21]. 


\section{MATERIALS AND METHODOLGY}

\section{Subjects}

As described elsewhere [20], subjects were 132 healthy adult male or female monozygotic twins aged 18-76 years who participated in the Tufts Twin Study. They included 60 monozygotic twin pairs who were either reared apart since near birth (22 pairs) or together (38 pairs), and 12 singleton monozygotic twins (3 MZA, 9 MZT) whose twin did not participate in the study. Eligibility criteria included being healthy at the time of study and willing to travel to Boston to participate in the study. Subjects were given a stipend for their participation in the study. The protocol was approved by the Institutional Review Board at Tufts University and all subjects gave written and informed consent.

\section{Protocol}

Each subject was admitted to the Metabolic Research Unit at the HNRCA for approximately four days of observation and completed a variety of examinations and questionnaires concerning energy regulation. During the 4-day inpatient study at Tufts, subjects slept and ate all meals in the research center from a self-selection menu of typical foods [20]. BMI was calculated from measured weight and height.

\section{Eating Behavior Measures}

Subjects completed the Three Factor Eating Questionnaire [18], which consists of 51 questions contributing towards the scoring of constructs and subscales. The questionnaire was completed with minimal environmental distractions and not in the presence of other people, including their twin.

As originally developed by Stunkard and Messick, the Three Factor Eating Questionnaire characterizes three main constructs of eating behavior - disinhibition, restraint and hunger [18]. Since the development of the questionnaire, ten subscales have been proposed [22, 23]. Descriptions of these constructs and subscales are presented in Appendix A.

Questionnaires from the 132 subjects were used to calculate the three original constructs and the ten subscales with scoring as described in the original reports. Of these questionnaires, 34 had some missing answers; in this case we used our previously developed method for scaling constructs and subscales [5]. When $<15 \%$ of the scale's questions were unanswered, proportional scales were calculated as [(raw score/ (total number of possible answers - number of missing answers)] $\times$ total number of possible answers). When $>15 \%$ of the scale's questions were unanswered, a score could not be calculated and data for the particular construct or subscale was considered missing. Appendix B summarizes our scoring calculations.

\section{Statistical Analysis}

As described elsewhere [20], descriptive statistics were calculated using SAS 9.1 [24]. Genetic and environmental influences on eating behavior were assessed using intrapair (intraclass) correlations and model-fitting analyses.
Intrapair (intraclass) MZA and MZT correlation coefficients were calculated for all constructs and subscales as a measure of within-pair similarity using SPSS 15.0 [25]. MZA intrapair correlations are a simple estimate of heritability, because their covariance consists solely of the genetic component of variance [26]. However, this technique of heritability estimation is inferior to model-fitting analyses because it may lead to nonsensical estimates of heritability, it does not easily generalize to the multivariate genetic factor models, it is inefficient when there are missing data, and it does not optimally combine data from multiple groups [27].

Model-fitting analyses are based on the decomposition of variance into genetic $(G)$, common or shared environmental $(C)$, and unique or non-shared environmental (E) components. Genetic variance $\left(\mathrm{V}_{\mathrm{G}}\right)$ is caused by differences in genes between individuals. The distinction between genetic variance due to dominance versus additive effects cannot be evaluated in an MZT/MZA study because both types share $100 \%$ of their genetic material, and therefore all additive and non-additive genetic variance components. Common environmental variance $\left(V_{C}\right)$ is due to environmental factors responsible for resemblance between family members, while unique environmental variance $\left(V_{E}\right)$ is due to environmental factors that contribute to differences between family members [26]. Unique environmental variance comprises any variance that is not due to genetic or common environmental factors, including variance due to measurement error. Total phenotypic variance $\left(V_{P}\right)$ can be represented as $V_{P}=V_{G}+$ $\mathrm{V}_{\mathrm{C}}+\mathrm{V}_{\mathrm{E}}$. Broad heritability is defined as the proportion of total phenotypic variance in a given trait that is attributable to genotypic variance, including all additive and nonadditive effects of genetic factors [26]. This quantity is distinct from the narrow sense heritability, which refers to only the additive genetic variance.

Variance decomposition was applied in the MZA/MZT design, where it is assumed that all monozygotic twin pairs share $100 \%$ of their genetic material. MZT pairs share all common environmental factors (such as parents, siblings, home, and economic factors), and MZAs do not correlate for these common environmental effects. That is, it is assumed that MZAs are placed in homes selected at random from the population. These relationships can be depicted in a path diagram (Fig. 1). The covariance of MZAs $\left(\mathrm{COV}_{\mathrm{MZA}}\right)$ is $\mathrm{V}_{\mathrm{G}}$ and the covariance of MZTs $\left(\mathrm{COV}_{\mathrm{MZT}}\right)$ is $\mathrm{V}_{\mathrm{G}}+\mathrm{V}_{\mathrm{C}}$ [20].

As described elsewhere, the MZA/MZT twin model used here is based on the following assumptions: (1) traits follow polygenic inheritance; (2) the observed phenotypic variance is a linear additive function of genetic and environmental variances; (3) genetic and environmental effects are uncorrelated and there is no genotype by environmental interaction; (4) there is no selective placement (non-random adoption of twins into similar families); (5) genetic and environmental effects are of the same magnitude in males and females $[20,28]$. Note also that any genetic effects of assortative mating contribute to $\mathrm{V}_{\mathrm{G}}$ and that differences in methylation within a twin pair contribute to $\mathrm{V}_{\mathrm{E}}$.

Model-fitting analyses were performed using $\mathrm{Mx}$, a structural equation modeling software package [28]. Mx fits the MZA/MZT GCE model to the raw observed data. It estimates parameters using maximum likelihood, and com- 


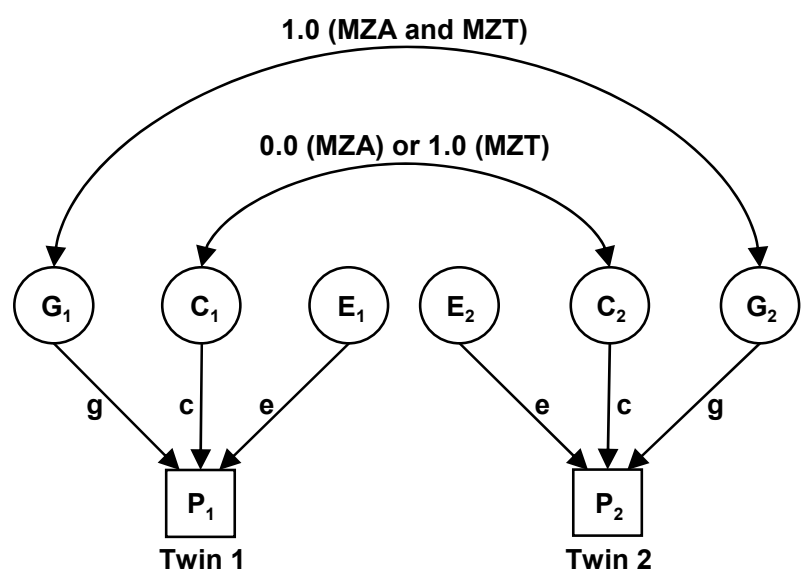

Fig. (1). Path diagram of the univariate MZA/MZT GCE twin model. MZA, monozygotic twins reared apart; MZT, monozygotic twins reared together; $\mathrm{G}$, genetic factors; $\mathrm{C}$, common environmental factors; E, unique environmental factors; g, c, e are path coefficients; $\mathrm{P}_{1}$, phenotype of twin $1 ; \mathrm{P}_{2}$, phenotype of twin 2. Circles represent latent (unmeasured) variables. Squares represent observed (measured) variables. Single-headed arrows represent hypothesized casual relationships between variables. Doubleheaded arrows represent correlation or covariance between variables [20].

putes goodness-of-fit statistics based on minus twice the natural logarithm of the likelihood (-2lnL). Likelihood ratio tests (LRT) are used to test hypotheses, because under certain regularity conditions, the difference in -2lnL between nested models (which differ because one or more parameters are constrained to equal each other or specific values) is asymptotically distributed as $\mathrm{chi}^{2}$ with degrees of freedom (df) equal to the difference in the number of free parameters in the two models. However, under the null hypothesis that a variance component is zero, the likelihood-ratio test is distributed as a 50:50 mixture of chi $^{2}$ with 1 degree of freedom, and zero.

Univariate analyses were performed to quantify and compare the genetic, common environmental and unique environmental influences on eating behavior constructs assessed by the Eating Inventory, including subscales of restraint, disinhibition, and hunger. All the subscales were treated as ordinal variables because they had less than ten distinct categories. Restraint, disinhibition and hunger which had greater than ten categories - were analyzed both as ordinal and continuous variables, to compare results. Age and gender were included as covariates.

Multivariate analysis was used to determine the extent to which genetic and environmental influences are shared by restraint, disinhibition, and hunger. Age and gender were again included in the analyses as covariates. Two multivariate models - a Cholesky decomposition and a common pathway multivariate model - were compared on the basis of likelihood and parsimony to determine the model with the best fit to the data. The difference in likelihood was assessed by calculating the difference in -2lnL between models. Parsimony was assessed by Akaike's Information Criterion (AIC), which may be computed as $-2 \ln L-2 \mathrm{df}$, where the more negative value indicated the most parsimonious model.
A Cholesky decomposition model is used to estimate the genetic and environmental covariances across the three variables: restraint, disinhibition, and hunger [29]. In this approach, the observed variables are influenced by three latent $\mathrm{G}$ factors, three latent $\mathrm{C}$ factors, and three latent $\mathrm{E}$ factors. The model is specified such that the first genetic factor influences all three variables, the second genetic factor influences the final two variables, and the third genetic factor influences only the last variable. The same pattern is specified for the common and unique environmental factors. This model is 'saturated' in that it estimates all genetic and environmental variances and covariances subject to the constraint that the matrices of these variance components are non-negative definite.

In the common pathway model, a common latent factor influences all of the observed variables; this common factor is in turn influenced by $\mathrm{G}, \mathrm{C}$ and $\mathrm{E}$ latent factors. In addition, there are three specific latent $G$ factors $\left(G_{S}\right)$, three specific latent $C$ factors $\left(C_{S}\right)$, and three specific latent $E$ factors $\left(E_{S}\right)$. These specific factors each affect only one observed variable.

\section{RESULTS}

Table 1 shows descriptive statistics of the study population. As described elsewhere [20], the majority of the subjects were female (72\% of MZAs and $76 \%$ of MZTs) and Caucasian (97\% of MZAs and 94\% of MZTs). The mean age of the MZA twins (49.1 \pm 12.0 years, range of 22-76 years) was significantly different from that of the MZT twins (28.7 \pm 7.3 years, range of $18-47$ years, $p<0.0001$ ). MZTs and MZAs differed significantly in weight and body mass index $(\mathrm{p}<0.01)$, but not when the data were adjusted for age ( $>0.05)$. Of all the eating behavior constructs, the two groups differed significantly only in restraint and two of the restraint subscales (flexible and rigid restraint), with MZTs scoring higher than MZAs $(\mathrm{p}<0.05)$. The number of subjects contributing to mean scores varies slightly between constructs and subscales because individual questions were occasionally left unanswered.

Table 2 shows the intrapair (intraclass) correlations for MZAs and MZTs. MZT correlations were greater than MZA correlations for restraint, disinhibition, attitude to selfregulation, habitual susceptibility, and flexible restraint, suggesting that common environmental factors play a role in these eating behaviors. MZA correlations are a simple estimate of heritability, showing significant estimates ranging from 0.47 (situational susceptibility) to 0.62 (avoidance of fattening foods) for the various eating behavior constructs. However, as mentioned earlier, this technique of heritability estimation is inferior to model-fitting analyses [27].

Results from the univariate analyses are shown in Fig. (2), with confidence intervals for variance component estimates reported in Table 3 . Results for restraint, disinhibition and hunger were similar when the constructs were analyzed as ordinal versus continuous variables, so only the results from the continuous variable analyses are presented. Only restraint, emotional and situational susceptibility to disinhibition, and internal locus for hunger had statistically significant heritability estimates, those being 52\%, 55\%, $38 \%$ and $50 \%$, respectively. The proportion of variance due 
Table 1. Characteristics of Study Population [20]

\begin{tabular}{|c|c|c|c|}
\hline & \multicolumn{2}{|c|}{ Mean \pm SD $\left(n^{\mathrm{a}}\right)$} & \multirow[b]{2}{*}{$P^{\mathbf{b}}$} \\
\hline & MZA & MZT & \\
\hline Age (years) & $50.7 \pm 10.4(47)$ & $28.7 \pm 7.7(85)$ & $<0.0001$ \\
\hline Height $(\mathrm{cm})$ & $166.6 \pm 9.8(47)$ & $169.7 \pm 7.6(85)$ & 0.0798 \\
\hline BMI $\left(\mathrm{kg} / \mathrm{m}^{2}\right)$ & $27.5 \pm 5.4(47)$ & $23.1 \pm 3.4(85)$ & $<0.0001^{\mathrm{C}}$ \\
\hline \multicolumn{4}{|c|}{ Eating Inventory Constructs (3) (possible scoring range in italics) } \\
\hline Disinhibition (0-16) & $5.8 \pm 3.6(45)$ & $5.6 \pm 3.3(84)$ & 0.7299 \\
\hline Hunger $(0-14)$ & $4.7 \pm 3.3(45)$ & $5.6 \pm 3.1(84)$ & 0.1517 \\
\hline \multicolumn{4}{|l|}{ Eating Inventory Subscales of Bond et al. (25) } \\
\hline \multicolumn{4}{|l|}{ Restraint } \\
\hline Strategic Dieting Behavior $(0-4)$ & $0.8 \pm 1.1(45)$ & $1.3 \pm 1.4(84)$ & 0.0539 \\
\hline Emotional Susceptibility (0-3) & $1.1 \pm 1.1(47)$ & $1.0 \pm 1.1(83)$ & 0.7983 \\
\hline Situational Susceptibility (0-5) & $2.2 \pm 1.6(47)$ & $2.2 \pm 1.3(85)$ & 0.8585 \\
\hline \multicolumn{4}{|l|}{ Hunger } \\
\hline Internal Locus for Hunger (0-6) & $1.9 \pm 1.8(46)$ & $2.5 \pm 1.9(85)$ & 0.0954 \\
\hline External Locus for Hunger (0-6) & $1.7 \pm 1.5(45)$ & $1.7 \pm 1.5(84)$ & 0.8849 \\
\hline \multicolumn{4}{|l|}{ Restraint Subscales of Westenhoefer (26) } \\
\hline Flexible Restraint (0-7) & $2.1 \pm 1.8(45)$ & $3.1 \pm 1.8(84)$ & 0.0132 \\
\hline Rigid Restraint (0-7) & $1.9 \pm 1.5(45)$ & $2.8 \pm 1.8(84)$ & 0.0300 \\
\hline
\end{tabular}

MZA, monozygotic twins reared apart; MZT, monozygotic twins reared together.

${ }^{a} \mathrm{n}$, number of individuals.

${ }^{\mathrm{b}} \mathrm{P}$ for statistical difference between MZA and MZT twins corrected for sampling among twins.

${ }^{\mathrm{c}}$ Differences between MZA and MZT means were not statistically significant when adjusting for age, age $\mathrm{e}^{2}$, and age $\mathrm{e}^{3}(P>0.05)$.

to common environmental factors was not statistically significant for any of the constructs or subscales, while the proportion of variance of constructs and subscales due to unique environmental factors (which is perforce statistically significant because the likelihood of the data approaches zero in the limit as the estimate of $\mathrm{E}$ does) ranged from $45 \%$ to $71 \%$.

Multivariate model-fitting analyses were also conducted on restraint, disinhibition and hunger (treated as continuous variables) to determine the extent to which they share genetic and environmental influences. Variances-covariance and correlation matrices for restraint, disinhibition, and hunger are shown in Table 4 . The difference in fit $(-2 \ln L)$ between the Cholesky decomposition and the common pathway model was 6.7 with 4 degrees of freedom. Since the p-value associated with this chi-square distribution is 0.152 , the two models do not significantly differ in fit, and therefore the simpler common pathway model is judged acceptable. Fig. (3) shows a path diagram for the common pathway model, with standardized parameter estimates and confidence intervals drawn along the paths and statistically significant paths represented as darkened lines. Disinhibition and hunger appeared to share a common influence, which was influenced (although not statistically significantly) by genetic and environmental factors. Specific genetic and unique environmental factors statistically significantly influenced restraint; specific environmental factors did not statistically signifi-

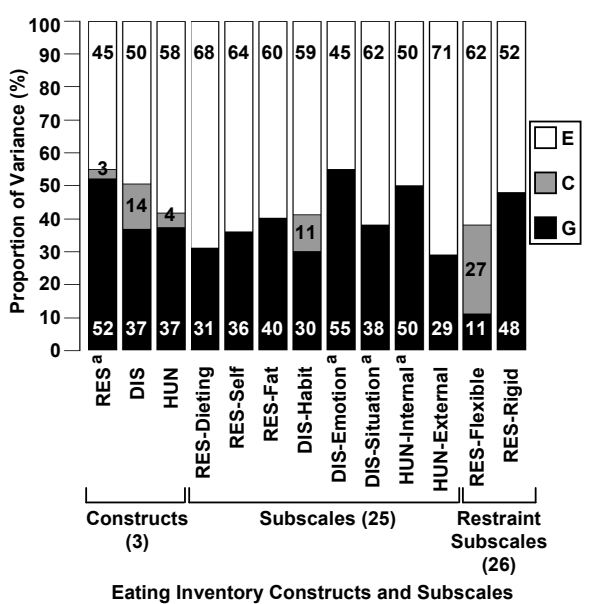

Fig. (2). Variance components from univariate GCE twin model for Eating Inventory constructs and subscales. G, genetic; C, common environmental; E, unique environmental; RES, restraint; Dieting, strategic dieting behavior; Self, attitude to self-regulation; Fat, avoidance of fattening foods; DIS, disinhibition; Habit, habitual susceptibility; Emotion, emotional susceptibility; Situation, situational susceptibility; HUN, hunger; Internal, internal locus; External, external locus.

All variables have non-statistically significant C components and statistically significant E components.

${ }^{a}$ Statistically significant G component. 
Table 2. Intrapair MZA and MZT Correlations

\begin{tabular}{|c|c|c|c|c|}
\hline & \multicolumn{2}{|r|}{ MZA } & \multicolumn{2}{|r|}{ MZT } \\
\hline & $\mathbf{n}^{\mathrm{a}}$ & Intrapair Correlation (95\% CI) & $\mathbf{n}^{\mathrm{a}}$ & Intrapair Correlation (95\% CI) \\
\hline Weight (kg) & 22 & $0.66(0.35,0.84)$ & 38 & $0.87(0.77,0.93)$ \\
\hline BMI $\left(\mathrm{kg} / \mathrm{m}^{2}\right)$ & 22 & $0.55(0.18,0.78)$ & 38 & $0.80(0.66,0.89)$ \\
\hline \multicolumn{5}{|l|}{ Eating Inventory Constructs (3) } \\
\hline Hunger & 20 & $0.42(-0.01,0.72)$ & 38 & $0.42(0.12,0.65)$ \\
\hline \multicolumn{5}{|l|}{ Eating Inventory Subscales of Bond et al. (25) } \\
\hline \multicolumn{5}{|l|}{ Restraint } \\
\hline Strategic Dieting Behavior & 20 & $0.35(-0.08,0.68)$ & 38 & $0.30(-0.02,0.56)$ \\
\hline Habitual Susceptibility & 20 & $0.32(-0.12,0.66)$ & 36 & $0.53(0.25,0.73)$ \\
\hline Emotional Susceptibility & 22 & $0.60(0.25,0.81)$ & 37 & $0.60(0.35,0.77)$ \\
\hline Situational Susceptibility & 22 & $0.47(0.07,0.74)$ & 38 & $0.38(0.08,0.62)$ \\
\hline \multicolumn{5}{|l|}{ Hunger } \\
\hline Internal Locus for Hunger & 21 & $0.58(0.21,0.80)$ & 38 & $0.41(0.11,0.64)$ \\
\hline External Locus for Hunger & 20 & $0.37(-0.07,0.69)$ & 38 & $0.25(-0.07,0.52)$ \\
\hline \multicolumn{5}{|l|}{ Restraint Subscales of Westenhoefer (26) } \\
\hline Flexible Restraint & 20 & $0.39(-0.04,0.71)$ & 37 & $0.49(0.21,0.70)$ \\
\hline Rigid Restraint & 20 & $0.58(0.21,0.81)$ & 37 & $0.57(0.31,0.75)$ \\
\hline
\end{tabular}

MZA, monozygotic twins reared apart; MZT, monozygotic twins reared together.

${ }^{a} \mathrm{n}$, number of twin pairs.

cantly influence disinhibition; and a unique environmental factor statistically significantly influenced hunger. The proportion of variance due to genetic and environmental factors for each observed measure is shown in the corresponding square in Fig. (3). Genetic variance in restraint was statistically significant with heritability estimated at $51 \%$. The variance of disinhibition and hunger was explained mostly by environmental factors, with non-statistically significant heritabilities of $34 \%$ and $36 \%$, respectively. These values differed slightly from the univariate estimates because multivariate analyses exploit additional data in the form of covariances between variables, both within-person and acrosstwins.

\section{DISCUSSION AND CONCLUSION}

This study of adult MZA and MZT pairs provides, to our knowledge, the first report of the genetic, common environmental and unique environmental influences on eating behavior subscales, and adds to the currently small existing literature on the heritability of the original three eating behavior constructs in a unique population recognized to have relatively little bias in estimates of heritability. The primary findings of this study were that, out of 14 constructs and subscales, only 4 were significantly heritable, and that unique environmental transmission was particularly high for the external locus for hunger and strategic dieting behavior of restraint. These findings suggest an important role for the environment in influencing eating behaviors that contribute to or maintain excess body fatness.

The four eating behavior constructs and subconstructs that were significantly heritable in this study were restraint, emotional susceptibility to disinhibition, situational susceptibility to disinhibition, and internal locus for hunger (heritabilities were $52 \%, 55 \%$, 38\% and 50\%, respectively), traits that have been associated with body fatness and/or weight gain [ $3,5,8,29,30]$. Concerning restraint, while previous investigations have indicated that dietary restraint in children is strongly influenced by parental behaviors $[9,10]$, heritability estimates in adults have ranged from $0 \%$ to $61 \%$ [1217] and our estimate of $52 \%$ is thus near the top end of the reported range. In part, the variability in previous studies may have been due to the different instruments and populations used. For example, classical twin studies using MZ and DZ twins may potentially overestimate heritability because they rely on the "equal environment assumption" which may be violated, though it seems unlikely that such violations do not originate with the phenotype or genotype of the twins. Family studies may alternatively underestimate heritability for a variety of reasons including false paternity [21] and genotype by age interaction, and in the absence of relationships of differing degree require the assumption that shared environment effects are absent. Consistent with the suggestion of less bias in an MZA/MZT population, our results 
Table 3. Variance Components from Univariate GCE Model for Eating Inventory Constructs and Subscales

\begin{tabular}{|c|c|c|c|}
\hline & \multicolumn{3}{|c|}{ Proportion of Variance (95\% CI) } \\
\hline Restraint & $0.52(0.10,0.69)$ & $0.03(0.00,0.48)$ & $0.45(0.28,0.67)$ \\
\hline Disinhibition & $0.37(0.00,0.62)$ & $0.14(0.00,0.61)$ & $0.50(0.31,0.76)$ \\
\hline \multicolumn{4}{|l|}{ Eating Inventory Subscales of Bond et al. (26) } \\
\hline \multicolumn{4}{|l|}{ Restraint } \\
\hline Strategic Dieting Behavior & $0.31(0.00,0.57)$ & $0.00(0.00,0.45)$ & $0.69(0.42,1.00)$ \\
\hline Attitude to Self-Regulation & $0.36(0.00,0.58)$ & $0.00(0.00,0.41)$ & $0.64(0.41,0.91)$ \\
\hline Avoidance of Fattening Foods & $0.40(0.00,0.64)$ & $0.00(0.00,0.48)$ & $0.60(0.34,0.92)$ \\
\hline Emotional Susceptibility & $0.55(0.11,0.75)$ & $0.00(0.00,0.49)$ & $0.45(0.23,0.71)$ \\
\hline Situational Susceptibility & $0.38(0.04,0.59)$ & $0.00(0.00,0.35)$ & $0.62(0.40,0.88)$ \\
\hline \multicolumn{4}{|l|}{ Hunger } \\
\hline Internal Locus for Hunger & $0.50(0.15,0.69)$ & $0.00(0.00,0.36)$ & $0.50(0.31,0.75)$ \\
\hline External Locus for Hunger & $0.29(0.00,0.52)$ & $0.00(0.00,0.42)$ & $0.71(0.46,0.98)$ \\
\hline \multicolumn{4}{|l|}{ Restraint Subscales of Westenhoefer (27) } \\
\hline Flexible Restraint & $0.11(0.00,0.50)$ & $0.27(0.00,0.62)$ & $0.62(0.37,0.94)$ \\
\hline Rigid Restraint & $0.48(0.00,0.67)$ & $0.00(0.00,0.54)$ & $0.52(0.30,0.77)$ \\
\hline
\end{tabular}

gave generally higher heritability estimates compared to the family studies [12, 15], and lower or non-significant estimates than the MZ/DZ studies [13, 14]. However, it should also be noted that our study population was not particularly large and the age range of the twins was wide, therefore some of the trends observed that did not reach statistical significance could have been due to lack of statistical power.

The ability to partition total variance into genetic, common environmental and unique environmental components by the use of structural equation modelling also showed that all eating behaviors studied were substantially influenced by the unique environment, a finding that is consistent with the increasing prevalence of obesity nationally and worldwide in parallel with changing environmental factors. In contrast to suggestions from two previous classical (MZ vs DZ) twin studies [13, 14], none of the eating behaviors measured in this study was significantly influenced by the common environment. In contrast, unique environmental factors appeared to have a dominant influence on most eating behavior constructs and subscales, and were particularly strong for habitual susceptibility to disinhibition, the external locus for hunger and the restraint subscales, all of which may potentially play an important role in excess weight gain in adulthood and the development of obesity. It is possible that we underestimated the influence of common environmental factors if our assumption of zero MZA common environmental covariance was not true due to selective placement of MZA

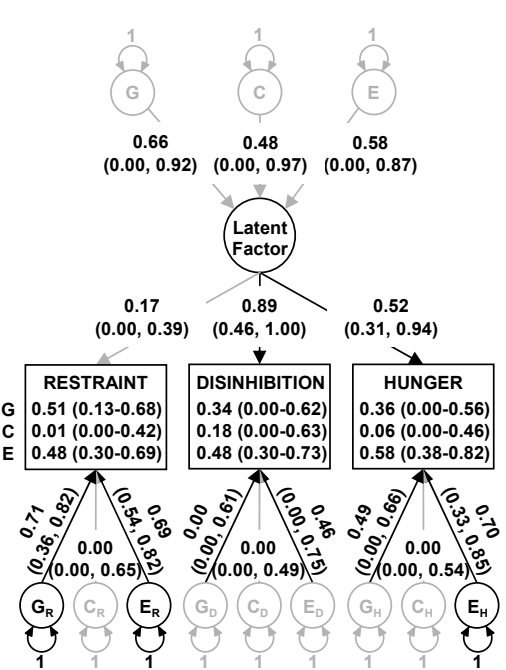

Fig. (3). Common pathway model path diagram of Eating Inventory constructs. G, genetic; C, common environmental; E, unique environmental. Rectangles represent observed variables, and the proportion of variance due to G, C and E (with 95\% CI in parentheses) is printed in each rectangle. Circles represent latent or unmeasured variables. Single-headed arrows represent hypothesized casual relationships between variables. Double-headed arrows represent variance. Path coefficients are standardized parameter estimates and 95\% confidence intervals are reported in parentheses. Darkened lines indicate statistically significant paths. Subscripts indicate variable or factor under influence. 
Table 4. Variance-covariance and Correlation Matrices of Eating Inventory Constructs for MZAs and MZTs

\begin{tabular}{|c|c|c|c|c|c|c|}
\hline \multicolumn{7}{|c|}{ Monozygotic Twins Reared Apart (MZA) } \\
\hline RES1 & 20.81 & -0.04 & -0.21 & 0.58 & -0.11 & -0.34 \\
\hline DIS1 & -0.77 & 14.22 & 0.35 & -0.09 & 0.40 & -0.16 \\
\hline HUN1 & -2.95 & 4.12 & 9.80 & 0.03 & 0.21 & 0.45 \\
\hline HUN2 & -5.25 & -2.05 & 4.55 & -2.77 & 3.77 & 11.37 \\
\hline \multicolumn{7}{|c|}{ Monozygotic Twins Reared Together (MZT) } \\
\hline Construct & RES1 & DIS1 & HUN1 & RES2 & DIS2 & HUN2 \\
\hline RES1 & 25.05 & 0.39 & 0.12 & 0.64 & 0.16 & 0.10 \\
\hline RES2 & 15.35 & 7.70 & 3.71 & 22.56 & 0.18 & -0.01 \\
\hline DIS2 & 2.77 & 6.29 & 2.98 & 2.72 & 10.50 & 0.57 \\
\hline HUN2 & 1.84 & 4.94 & 4.13 & -0.09 & 6.30 & 11.73 \\
\hline
\end{tabular}

Variances are on the leading diagonal, covariances are below the diagonal, and correlations are above the diagonal. RES, restraint; DIS, disinhibition; HUN, hunger. Numbers at the end of the variable name indicate twin 1 or twin 2 .

twin pairs into adoptive families that were similar to one another. However, selective placement is not thought to contribute significantly to MZA common environmental covariance [31]. Furthermore, in addition to the fact that a dominant role for unique environmental influences being entirely consistent with the changing national prevalence of obesity, it is also important to note that unique environment factors as assessed in these analyses include wide variety of influences including the current food environment of the individual which may have an important effect as well as factors specific to individuals during child-rearing years (for example, differing school meal environments), environmental influences after leaving home, and measurement error.

In a multivariate analysis, we also found that disinhibition and hunger are related to each other but unrelated to restraint - a finding that is consistent with a previous report [13]. A relationship between disinhibition and hunger is also consistent with previous work from our group, which showed both an interaction between habitual disinhibition and external hunger in eating behavior models predicting adult weight gain [8], and an association between food cravings (which are associated with disinhibition) and hunger [32]. The underlying reasons for disinhibition and hunger may be linked metabolically are not known, but we speculate that one candidate mechanism may be the cephalic phase of digestion [33,34], which occurs when the sympathetic nervous system is stimulated by sensory input on available food or thoughts about food and causes physiologic changes including: increased saliva production, increased gastric acid secretions and mobility, and an insulin surge and drop in blood glucose that can increase hunger [35]. As demonstrated by Pavlov's original experiments on associative conditioning in dogs [33], cephalic phase responses to food can be triggered by external factors if food consumption and the external factors occur together over repeated feeding bouts. Based on this premise, a habitual environment favorable for unrestricted eating could lead to high values for both habitual disinhibition and external hunger mediated by cephalic phase anticipatory responses to eating. Further research on the role of the environment in conditioning hunger and disinhibition is needed.

In summary, our results from a unique MZA/MZT population suggest a relatively modest influence of genes and a strong influence of unique environmental factors on eating behaviors. Although studies of this kind do not directly address whether the heritability of eating behaviors reflects the extent to which they can be changed with behavioral weight control or other therapies, our results suggest that environmental factors generally play a substantial role in individual differences in eating behaviors.

\section{CONFLICT OF INTEREST}

None declared.

\section{ACKNOWLEDGEMENTS}

We thank the volunteers who participated in this study, Angela Vinken for her assistance with data collection, Brenda Roche for her assistance in data preparation, and Dr. Gerard E. Dallal and Benjamin M. Neale for their statistical assistance during manuscript preparation.

The authors' responsibilities were as follows - SJE: analyzed the data and wrote the manuscript, which was reviewed and modified by all authors; MCN: provided guidance regarding the experimental design and statistical analyses; PJF: coordinated the study; AHL: provided guidance regarding data analysis and manuscript goals; ASG and ES: participated in data collection and medical supervision of the 
study; MAM: performed preliminary data cleaning and analyses; TJB: provided assistance in subject recruitment and guidance in study design; SBR: designed and directed all aspects of the study and contributed to the manuscript. None of the authors had a personal or financial conflict of interest.

Funding support for this project was provided by NIH grants DK046124 (all), 5T32 HL069772 (SJE), MH65322 APPENDICES

Appendix A: Description of Eating Inventory Constructs and Subscales
(MCN), DA18673 (MCN), DK73321 (AHL), and the U.S. Department of Agriculture under agreement no. 1950-51000061-04S (SJE, PJF, MAM, ES, SBR), 1950-51000-059-04S (AHL) and 1950-51000-061-05S (ASG). Any opinions, findings, conclusions, or recommendations expressed in this publication are those of the authors and do not necessarily reflect the view of the U.S. Department of Agriculture.

\begin{tabular}{|c|c|}
\hline \multicolumn{2}{|l|}{ Eating Inventory Constructs [3] } \\
\hline Restraint & $\begin{array}{l}\text { Conscious restriction of energy intake to prevent weight gain or promote weight loss } \\
\text { by, for example, counting calories and purchasing low calorie food items }\end{array}$ \\
\hline Disinhibition & Tendency to overeat in response to different stimuli and triggers \\
\hline Hunger & $\begin{array}{l}\text { Susceptibility to eat in response to perceived physiological symptoms that signal the } \\
\text { need for food }\end{array}$ \\
\hline \multicolumn{2}{|c|}{ Eating Inventory Subscales of Bond et al. [25] } \\
\hline \multicolumn{2}{|l|}{ Restraint } \\
\hline Strategic Dieting Behavior & $\begin{array}{l}\text { Behaviors that might be used to control weight (e.g. deliberately taking small help- } \\
\text { ings) }\end{array}$ \\
\hline Attitude to Self-Regulation & $\begin{array}{l}\text { Overarching perspective on eating and weight control (e.g. feeling that life is too short } \\
\text { to worry about dieting) }\end{array}$ \\
\hline Avoidance of Fattening Foods & Dieting behavior which limits calorie-dense foods \\
\hline \multicolumn{2}{|l|}{ Disinhibition } \\
\hline Habitual Susceptibility & Recurrent disinhibition triggered by routine circumstances \\
\hline Emotional Susceptibility & Disinhibition associated with negative affective states \\
\hline Situational Susceptibility & Disinhibition initiated by specific environmental cues (e.g. social occasions) \\
\hline \multicolumn{2}{|l|}{ Hunger } \\
\hline Internal Locus for Hunger & Hunger that is interpreted and regulated internally \\
\hline External Locus for Hunger & Hunger that is triggered by external cues \\
\hline \multicolumn{2}{|c|}{ Restraint Subscales of Westenhoefer [26] } \\
\hline Flexible Restraint & $\begin{array}{l}\text { An adaptable approach to dieting, where forbidden foods can be eaten in limited } \\
\text { quantities without guilt }\end{array}$ \\
\hline Rigid Restraint & $\begin{array}{l}\text { A dichotomized "all-or-nothing" approach to eating and dieting, where individuals } \\
\text { diet frequently and avoid forbidden foods, but exhibit behaviors that do not support } \\
\text { their dieting goals (e.g. not reducing meal size, not compensating for deviations from } \\
\text { the diet) }\end{array}$ \\
\hline
\end{tabular}

\section{Appendix B: Eating Inventory and Scoring Summary}

\section{Eating Inventory Scoring Summary}

The Eating Inventory consisted of 51 questions that contributed to the scoring of eating behavior constructs and subscales. Each question contributed to the score of one of the three original Eating Inventory constructs (restraint, disinhibition, and hunger), and most questions contributed to the score of one or more Eating Inventory subscales, as shown in the following table and questionnaire. Questions were each worth one point; questionnaire answers in bold type accrued one point and answers in regular type accrued zero points. Higher scores indicate greater levels of the eating behavior. If answers were missing, proportional scales were calculated when less than $15 \%$ of the scale's questions were unanswered (7). The proportional scale was calculated as: 
raw score

total number of possible answers - number of missing answers $\times$ total number of possible answers

When greater than $15 \%$ of the scale's questions were unanswered, a score could not be calculated and was considered missing data.

Questionnaire

Construct or Subscale

Eating Inventory Constructs (3)

Restraint

Disinhibition

Hunger

\section{Scoring}

Range Questions

Eating Inventory Subscales of Bond et al. (25)

Restraint

Strategic Dieting Behavior

Attitude to Self-Regulation

Avoidance of Fattening Foods

Disinhibition

Habitual Susceptibility

Emotional Susceptibility

Situational Susceptibility

Hunger

Internal Locus for Hunger

External Locus for Hunger

Restraint Subscales of Westenhoefer (26)

Flexible Restraint

Rigid Restraint
$0-21$

0-16

$0-14$
$4,6,10,14,18,21,23,28,30,32,33,35,37,38,40$, $42,43,44,46,48,50$

$1,2,7,9,11,13,15,16,20,25,27,31,36,45,49,51$,

$3,5,8,12,17,19,22,24,26,29,34,39,41,47$
$0-4$

$0-5$

$0-4$

$0-5$

$0-3$

$0-5$

$0-6$

$0-6$

$0-7$

$0-7$
6, 23, 28, 48

$10,21,30,32,37$

$33,42,43,44$

11, 36, 45, 49, 51

$9,20,27$

$2,7,13,15,16$

3, 5, 12, 24, 34, 398 ,

$19,22,26,41,47$

$4,6,18,28,35,42,48$

$14,32,37,38,40,43,44$

Eating Inventory

Construct Subscale Question

D none 1. When I smell a sizzling steak or see a juicy piece of meat, I find it very difficult to keep from eating, even if I have just finished a meal.

2. I usually eat too much at social occasions, like parties and picnics.

3. I am usually so hungry that I eat more than three times a day.

5. Dieting is so hard for me because I just get too hungry.

H-I

6. I

$\mathrm{R}$

$\mathrm{R}-\mathrm{D}, \mathrm{R}-\mathrm{F}$

7. Sometimes things just taste so good that I keep on eating even when I am no longer hungry.

$\mathrm{H} \quad \mathrm{H}-\mathrm{E}$

8. Since I am often hungry, I sometimes wish that while I am eating, an expert would tell me that I have had enough or that I can have something more to eat.

D D-E

9. When I feel anxious, I find myself eating.

$\mathrm{R} \quad \mathrm{R}-\mathrm{S} \quad 10$. Life is too short to worry about dieting.

11. Since my weight goes up and down, I have gone on reducing diets more than once.
T F

T F

T F

T F

T F

T F

TF

TF

TF

TF D D-H

T F 


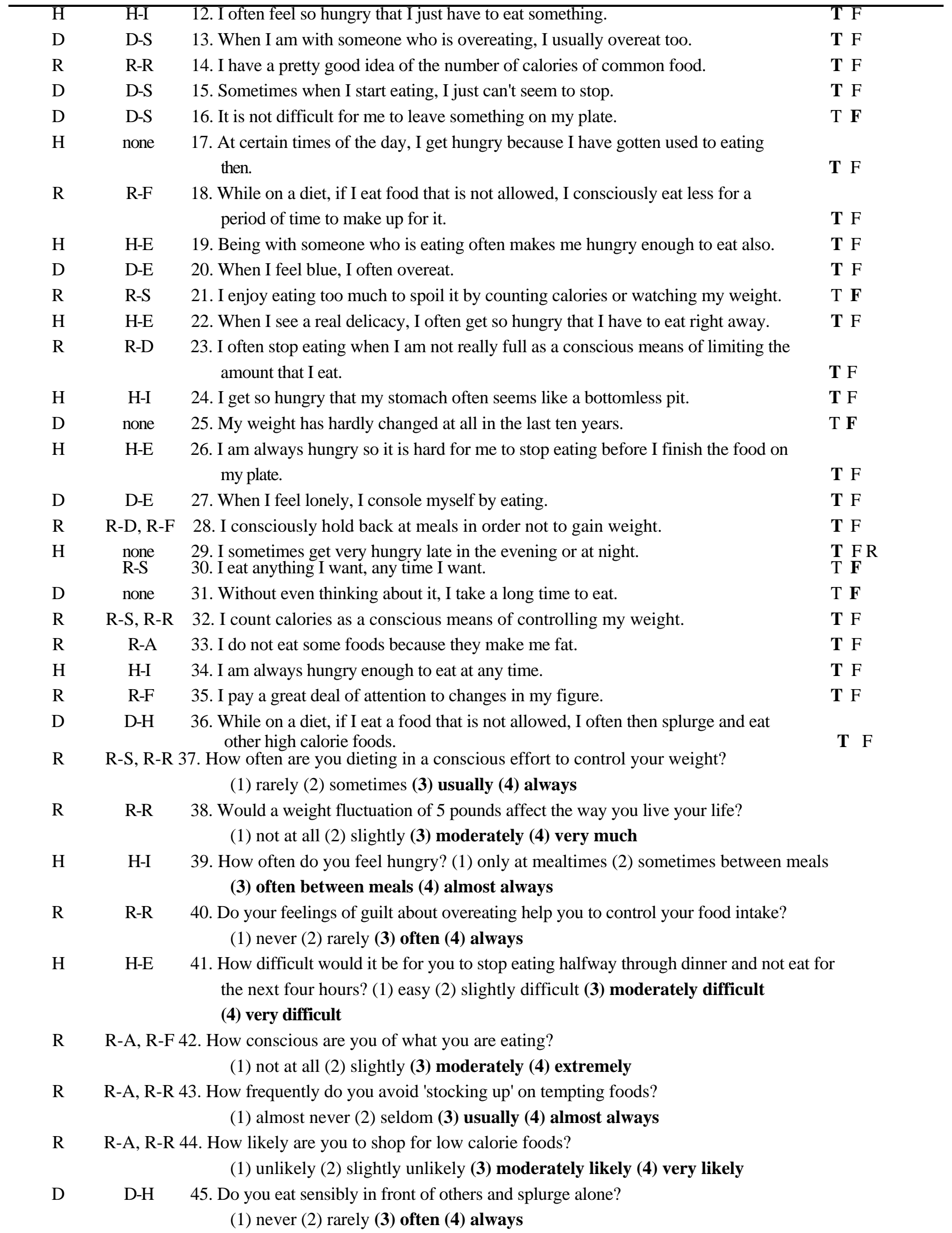


$\mathrm{R} \quad$ none 46. How likely are you to consciously eat slowly in order to cut down on how much you

(1) unlikely (2) slightly likely (3) moderately likely (4) very likely

$\mathrm{H} \quad \mathrm{H}-\mathrm{E} \quad$ 47. How frequently do you skip dessert because you are no longer hungry?

(1) almost never (2) seldom (3) at least once a week (4) almost every day

R R-D, R-F 48. How likely are you to consciously eat less than you want?

(1) unlikely (2) slightly likely (3) moderately likely (4) very likely

D D-H 49. Do you go on eating binges though you are not hungry?

(1) never (2) rarely (3) sometimes (4) at least once a week

$\mathrm{R} \quad$ none 50 . On a scale of 0 to 5 , where 0 means no restraint in eating (eating whatever you want, whenever you want it) and 5 means total restraint (constantly limiting food intake and never 'giving in'), what number would you give yourself? (Check

one.)

(0) eat whatever you want, whenever you want it

(1) usually eat whatever you want, whenever you want it

(2) often eat whatever you want, whenever you want it

(3) often limit food intake, but often 'give in'

(4) usually limit food intake, rarely 'give in'

(5) constantly limiting food intake, never 'give in'

D D-H 51. To what extent does this statement describe your eating behavior? 'I start dieting

in the morning, but because of any number of things that happen during the day, by evening I have given up and eat what I want, promising myself to start dieting

again tomorrow.'

(1) not like me (2) little like me (3) pretty good description of me

\section{(4) describes me perfectly}

Eating Inventory Constructs: $\mathrm{R}$ = restraint, $\mathrm{D}$ = disinhibition, $\mathrm{H}=$ hunger

Restraint Subscales: R-D = strategic dieting behavior, R-S = attitude to self-regulation, R-A = avoidance of fattening foods, R-F = flexible restraint, R-R = rigid restraint

Disinhibition Subscales: D-H = habitual susceptibility, D-E = emotional susceptibility, D-S = situational susceptibility

Hunger Subscales: H-I = internal locus for hunger, $\mathrm{H}-\mathrm{E}=$ external locus for hunger

Answers in bold type accure one point. Answers in regular type accrue zero points.

\section{REFERENCES}

[1] de Castro JM. Eating behavior: lessons from the real world of humans. Nutrition 2000; 16: 800-13.

[2] Lawson OJ, Williamson DA, Champagne CM, et al. The association of body weight, dietary intake, and energy expenditure with dietary restraint and disinhibition. Obes Res 1995; 3: 153-61.

[3] Williamson DA, Lawson OJ, Brooks ER, et al. Association of body mass with dietary restraint and disinhibition. Appetite 1995; 25: 3141.

[4] McGuire MT, Wing RR, Klem ML, Lang W, Hill JO. What predicts weight regain in a group of successful weight losers? J Consult Clin Psychol 1999; 67: 177-85.

[5] Hays NP, Bathalon GP, McCrory MA, Roubenoff R, Lipman R, Roberts SB. Eating behavior correlates of adult weight gain and obesity in healthy women aged 55-65 y. Am J Clin Nutr 2002; 75: 476-83.

[6] Provencher V, Drapeau V, Tremblay A, Despres JP, Lemieux S. Eating behaviors and indexes of body composition in men and women from the Quebec family study. Obes Res 2003; 11: 783-92.

[7] Bellisle F, Clement K, Le Barzic M, Le Gall A, Guy-Grand B, Basdevant A. The Eating Inventory and body adiposity from leanness to massive obesity: a study of 2509 adults. Obes Res 2004; 12 : 2023-30.

[8] Hays NP, Roberts SB. Aspects of eating behaviors "disinhibition" and "restraint" are related to weight gain and BMI in women. Obesity 2008; 16: 52-8.

[9] Carper JL, Orlet Fisher J, Birch LL. Young girls' emerging dietary restraint and disinhibition are related to parental control in child feeding. Appetite 2000; 35: 121-9.
[10] Francis LA, Birch LL. Maternal influences on daughters' restrained eating behavior. Health Psychol 2005; 24: 548-54.

[11] Savage JS, Fisher JO, Birch LL. Parental influence on eating behavior: conception to adolescence. J Law Med Ethics 2007; 35: 2234.

[12] Steinle NI, Hsueh WC, Snitker S, et al. Eating behavior in the Old Order Amish: heritability analysis and a genome-wide linkage analysis. Am J Clin Nutr 2002; 75: 1098-1106.

[13] Neale BM, Mazzeo SE, Bulik CM. A twin study of dietary restraint, disinhibition and hunger: an examination of the eating inventory (three factor eating questionnaire). Twin Res 2003; 6: 4718.

[14] de Castro JM, Lilenfeld LR. Influence of heredity on dietary restraint, disinhibition, and perceived hunger in humans. Nutrition 2005; 21: 446-55.

[15] Provencher V, Perusse L, Bouchard L, et al. Familial resemblance in eating behaviors in men and women from the Quebec Family Study. Obes Res 2005; 13: 1624-9.

[16] Tholin S, Rasmussen F, Tynelius P, Karlsson J. Genetic and environmental influences on eating behavior: the Swedish Young Male Twins Study. Am J Clin Nutr 2005; 81: 564-9.

[17] Keskitalo K, Tuorila H, Spector TD, et al. The Three-Factor Eating Questionnaire, body mass index, and responses to sweet and salty fatty foods: a twin study of genetic and environmental associations. Am J Clin Nutr 2008; 88: 263-71.

[18] Stunkard A, Messick S. The three-factor eating questionnaire to measure dietary restraint, disinhibition, and hunger. J Psychosom Res 1985; 29: 71-83.

[19] Rankinen T, Bouchard C. Genetics of food intake and eating behavior phenotypes in humans. Annu Rev Nutr 2006; 26: 413-34. 
[20] Elder JS, Lichtenstein AH, Pittas AG, et al. Genetic and environmental influences on factors associated with cardiovascular disease and the metabolic syndrome. J Lipid Res 2009; 50: 1917-26

[21] Allison DB, Kaprio J, Korkeila M, Koskenvuo M, Neale MC, Hayakawa K. The heritability of body mass index among an international sample of monozygotic twins reared apart. Int J Obes Relat Metab Disord 1996; 20: 501-6.

[22] Bond MJ, McDowell AJ, Wilkinson JY. The measurement of dietary restraint, disinhibition and hunger: an examination of the factor structure of the Three Factor Eating Questionnaire (TFEQ). Int J Obes Relat Metab Disord 2001; 25: 900-6.

[23] Westenhoefer J. Dietary restraint and disinhibition: is restraint a homogeneous construct? Appetite 1991; 16: 45-55.

[24] Inc. SI. SAS/STAT 9.1 User's Guide. Cary, NC: SAS Institute Inc.; 2004.

[25] Inc. S. SPSS 15.0 Brief Guide. Chicago, IL: SPSS, Inc.; 2006.

[26] Plomin R, DeFries JC, McClearn GE, Rutter M. Behavioral Genetics. $3^{\text {rd }}$ ed. New York: W.H. Freeman and Company 1997.

[27] Neale MC. Twin studies: software and algorithms. In: Cooper DN, Ed. Encyclopedia of the human genome. London: Macmillan Publishers Ltd, Nature Publishing Group 2003.
[28] Neale MC. Twin analysis. In: Armitage P, Colton T, Eds. Encyclopedia of biostatistics. New York: John Wiley 1998.

[29] Westenhoefer J, Pudel V, Maus N. Some restrictions on dietary restraint. Appetite 1990; 14: 137-41; discussion 142-3.

[30] Hays NP, Bathalon GP, Roubenoff R, McCrory MA, Roberts SB. Eating behavior and weight change in healthy postmenopausal women: results of a 4-year longitudinal study. J Gerontol 2006; 61A: 608-15.

[31] Plomin R, Loehlin JC. Direct and indirect IQ heritability estimates: a puzzle. Behav Genet 1989; 19: 331-42.

[32] Gilhooly CH, Das SK, Golden JK, et al. Food cravings and energy regulation: the characteristics of craved foods and their relationship with eating behaviors and weight change during 6 months of dietary energy restriction. Int J Obes 2007; 31: 1849-58.

[33] Pavlov IP. The work of the digestive glands. London: Charles Griffin Co. Ltd., 1902.

[34] Powley TL. The ventromedial hypothalamic syndrome, satiety, and a cephalic phase hypothesis. Psychol Rev 1977; 84: 89-126.

[35] Power ML, Schulkin J. Anticipatory physiological regulation in feeding biology: cephalic phase responses. Appetite 2008; 50: 194206.

(C) Elder et al.; Licensee Bentham Open

This is an open access article licensed under the terms of the Creative Commons Attribution Non-Commercial License (http://creativecommons.org/licenses/by-nc/3.0/) which permits unrestricted, non-commercial use, distribution and reproduction in any medium, provided the work is properly cited. 